\title{
Chimpanzees balance resources and risk in a human-influenced landscape of fear
}

\author{
Elena Bersacola ${ }^{1}$, Catherine Hill $^{2}$, and Kimberley Hockings ${ }^{1}$ \\ ${ }^{1}$ University of Exeter \\ ${ }^{2}$ Oxford Brookes University
}

November 17, 2020

\begin{abstract}
Coexistence between humans and wildlife is possible when animals are able to meet their ecological requirements while managing human-induced risks. Other than large carnivores, examination of fine-scale spatiotemporal interactions with humans have rarely been applied to threatened wildlife such as great apes, whose conservation relies on persistence in dynamic, shared landscapes. Using a landscape of fear framework with Bayesian INLA spatiotemporal modelling we investigate risk-mitigation and optimal foraging trade-offs in western chimpanzees (Pan troglodytes verus). Although humans and chimpanzees used the same locations within the agroforest landscape, chimpanzee space use was negatively mediated by villages and agriculture. However, chimpanzees responded to wild fruit scarcity by intensifying their use of village areas with cultivated fruits. Our data demonstrate dynamic spatiotemporal interactions in shared landscapes. An INLA-based landscape of fear approach generates a clear model output to examine risk mitigation/optimal foraging strategies, that can inform conservation interventions to promote human-wildlife coexistence.
\end{abstract}

\section{Hosted file}

Bersacola et al Ecology Letters MAIN TEXT.pdf available at https://authorea.com/users/376549/ articles/493479-chimpanzees-balance-resources-and-risk-in-a-human-influenced-landscapeof-fear 


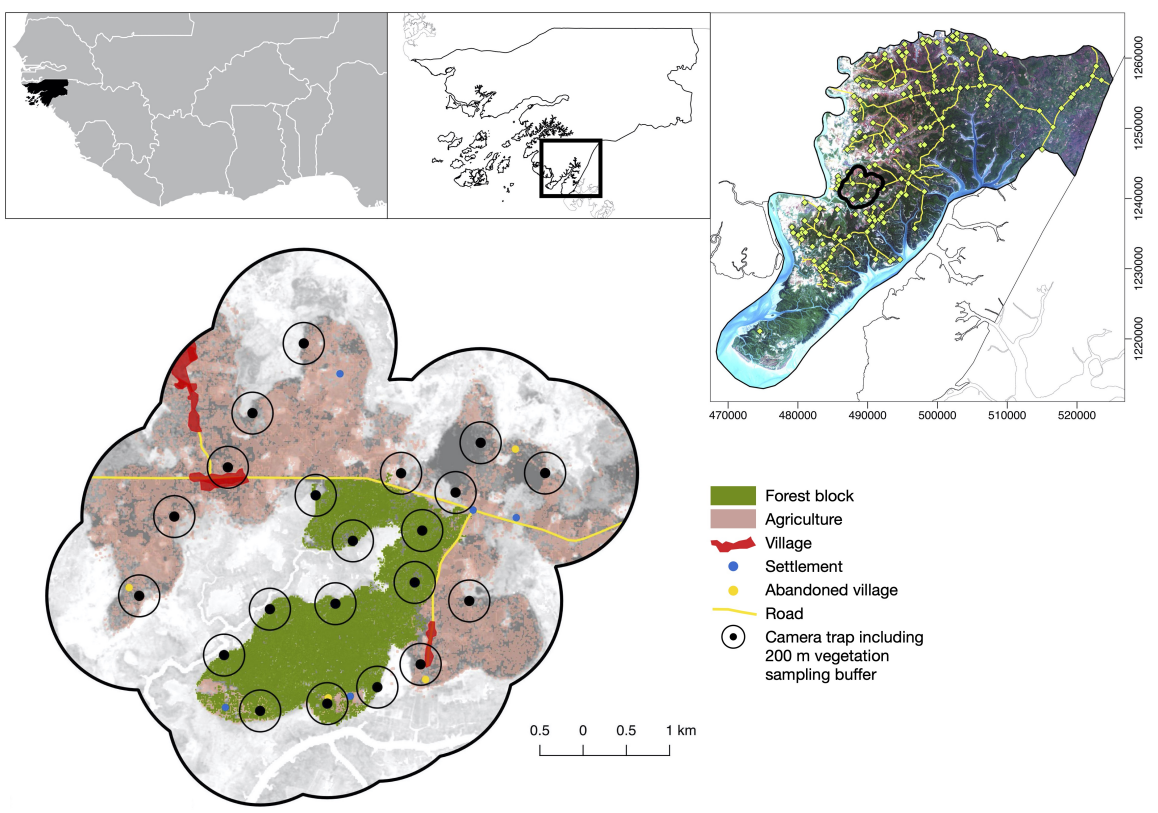

(a)

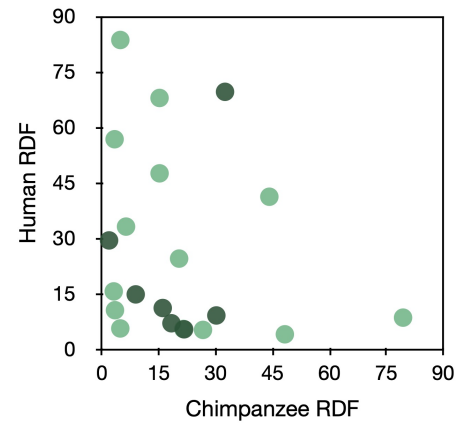

- Forest block - Matrix

- Chimpanzee ... Human
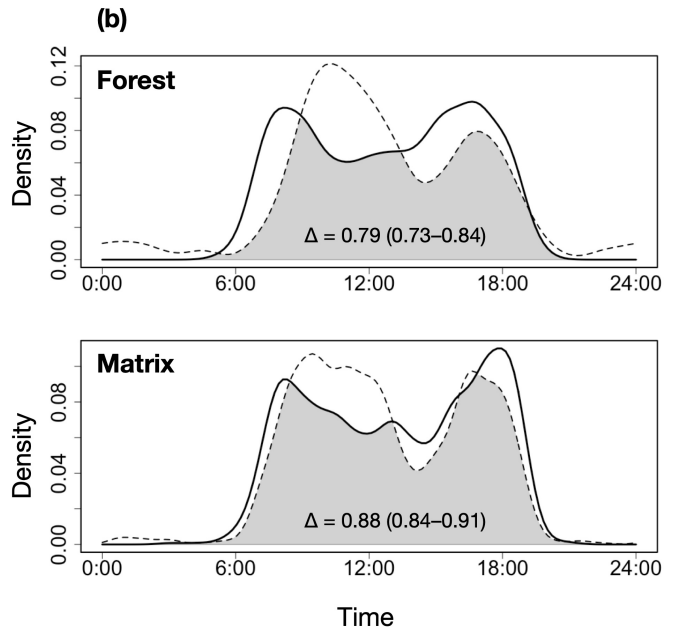


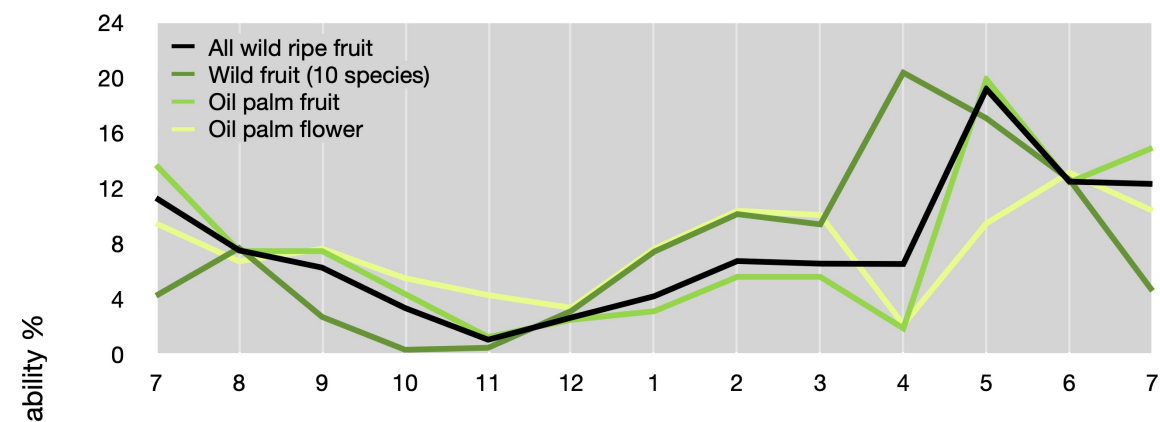

离
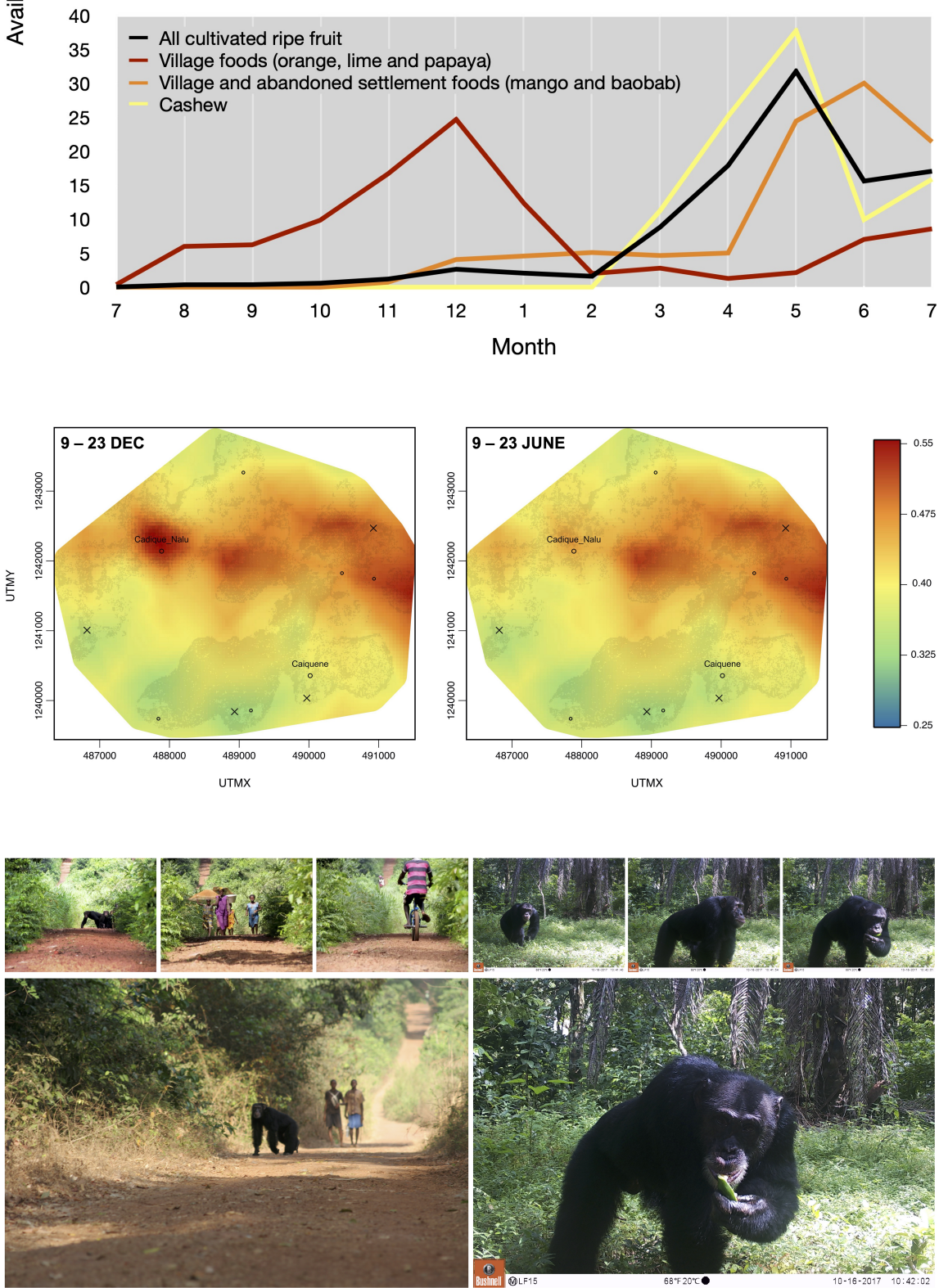